\title{
Surface treatment of gate insulating film for organic thin film transistor in plasma nitridation treatment
}

\author{
[ ${ }^{\circ}$ Akihito Matsushita, Keiichirou Minakuchi, Kabuto Hori, Yoshitaka Iwazaki, Tomo Ueno]
}

\begin{abstract}
The organic thin film transistor (OTFT) has been attractive as soft material for IoT devices, however there are some problems to overcome. One is the exitance of carrier traps at the interface between the organic semiconductor and the oxide film of the OTFT. The cause of this carrier trap is the presence of $\mathrm{OH}$ groups generated by the adsorption of water molecules in the air and $O$ atoms on the surface of the oxide film. As a method for removing this $\mathrm{OH}$ group, generally, the formation of SAM films on the oxide layer has been attempted, but there are disadvantages such as requiring dense film forming conditions and spending a long time for the film formation. In this study, we have tried to remove $\mathrm{OH}$ group and suppress the generation of additional carrier trap by surface treatment of gate insulation film in plasma nitridation process. From the results of TDS measurement and $\mathrm{C}-\mathrm{V}$ measurement, it was confirmed that $\mathrm{OH}$ group on the surface of the gate insulating film can be removed and additional $\mathrm{OH}$ group formation can be suppressed. Improvement of transistor characteristics by plasma nitridation treatment was also confirmed in I-V measurement. As a result, it was confirmed that plasma nitridation treatment is an effective means as the surface treatment method for the gate insulating films.
\end{abstract}

\section{Introduction}

\section{A. Structure of TFT}

Thin film transistor (TFT) is usually used as switching element for display device such as liquid crystal display (LCD), organic light emitted diode (OLED), and so on. As shown in fig.1, the principle of switching operation of TFT is primary due to applying gate voltage, which control the electrical properties of the semiconductor surface. When the gate voltage is applied, electrons or holes, injected from the source electrode, accumulated at the interface between the semiconductor and the gate insulator to form a channel region and they would conduct between the source and the drain due to the drain bias $\mathrm{V}_{\mathrm{D}}$. On the other hand, when no gate voltage is applied, no channel region has been generated so that no conduction occurs between source and drain.

- ${ }^{\circ}$ Akihito Matsushita, Keiichirou Minakuchi, Kabuto Hori, Yoshitaka Iwazaki, Tomo Ueno

- Tokyo univ. of agri. and tech.

- Japan

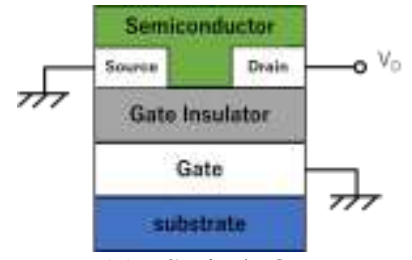

(a) Switch OFF

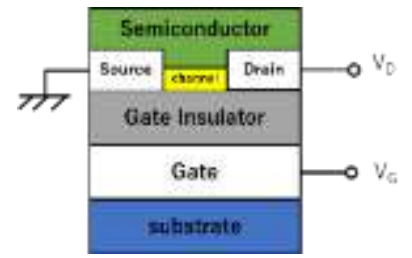

(b) Switch ON
Fig.1 Operating principle of TFT

There are four types of TFT structures shown in fig.2. The bottom gate structure as shown in figs. 2 (a) and (b) has an advantage that the heat during the insulating film formation does not affect the semiconductor layer. However, since the semiconductor layer is formed directly on the insulating film, the properties such as roughness and surface energy of the surface of the insulating film affect the semiconductor layer. In the top gate structure of (c) and (d), since the insulating film is formed after the formation of the semiconductor layer, there is concern that the heat at the time of forming the insulating film affects the semiconductor. In the bottom contact structure, shown in figs. 2 (b) and (d), on the other hand, since the semiconductor layer is formed by film formation of the source electrode and the gate electrode by photo lithography or so on, the process is simple and great industrial advantages are obtained. Therefore, in this research, a bottom gate / bottom contact type was adopted, and investigation was carried out.

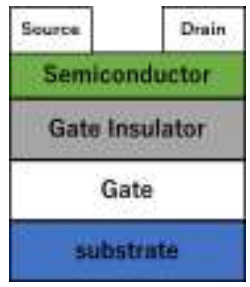

(a) Bottom gate $\cdot$ Top

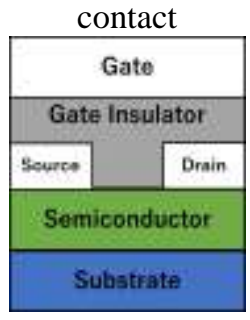

(c) Top gate $\cdot$ Top contact

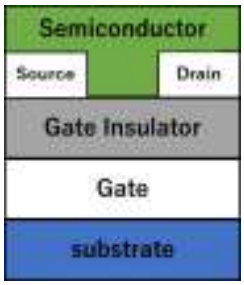

(b) Bottom gate $\cdot$ Bottom

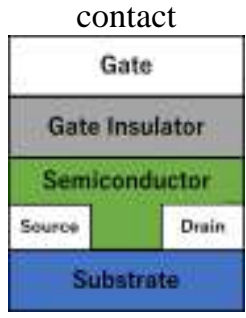

(d) Top gate $\cdot$ Bottom contact
Fig.2 Structure of TFT 


\section{B. Problems and solutions in OTFT structure fabrication}

In this study, organic thin film transistor (OTFT) using tetracene for semiconductor layer has been fabricated. Organic semiconductor materials have advantageous such as easy control of electrical characteristics and processing characteristics by chemical design and synthesis, compatibility with flexible devices due to the possibility of processing at low temperature, and so on. On the other hand, lower mobility compared to non-organic semiconductor materials such as $\mathrm{Si}$, or Ge is one of the problems to overcome for the practical device application.

The surface layer of the oxide film reacts with moisture in the air, that would be interface layer between the semiconductor layer and the gate insulator layer of the OTFT, whereby an $\mathrm{OH}$ group is present. This $\mathrm{OH}$ group serves as a carrier trap, resulting in further lowering of mobility and instability of operation.

One of the candidates for removing this carrier trap, a selfassembled monolayer (SAM film) has been proposed ${ }^{[1]}$. In this method, traps on the surface of the oxide film are removed and the surface energy is controlled by modifying the surface of the oxide film with densely adjusted organic molecules. However, as a problem of this method, it takes a long time and the severity of the film forming conditions to fabricate the SAM film. In this research, we propose plasma nitridation of the gate insulator surface.

In the plasma nitridation process carried out in this study, excited Ar by microwave irradiation is mixed with $\mathrm{N}_{2}, \mathrm{~N}_{2}$ is dissociated and/or excited to higher energy levels with high reactivity, which react with the surface of the oxide film to remove the $\mathrm{OH}$ group at the surface of the gate insulator film and form a nitride film layer at the surface.

\section{Experimental}

$\mathrm{n}^{+}$-Si (100) wafers with the resistivity of $0.014-0.017$ $[\Omega \cdot \mathrm{m}]$ has been used as the substrate. After the conventional chemical cleaning followed by dilute HF dip to remove the native oxide film, thermal oxidation is performed at $900\left[{ }^{\circ} \mathrm{C}\right]$ for $60[\mathrm{~min}]$, and an oxide film of about 20 [nm] thick is formed. Some of the samples of $\mathrm{SiO}_{2} / \mathrm{Si}$ structure have been air exposed for 1 day to characterize the effects of adsorbed $\mathrm{OH}$ groups on the $\mathrm{SiO}_{2}$ surface. Plasma treatment is performed for 30 [min] at $200\left[{ }^{\circ} \mathrm{C}\right.$ ] in $\mathrm{N}_{2}: \mathrm{Ar}=1: 100$ with 1 [Torr]. In order to investigate the improvement of moisture resistance, air exposure was carried out for one day after plasma treatment. Then, $\mathrm{Al}$ was deposited as a back contact, $\mathrm{Au}$ as a top contact, and tetracene as an organic semiconductor by vacuum evaporation to prepare an MOS structure of Fig.3 and a bottom contact type OTFT has been fabricated. Also, the amount of $\mathrm{OH}$ groups after each air exposure was evaluated by TDS measurement.
Table. 1 Experimental condition

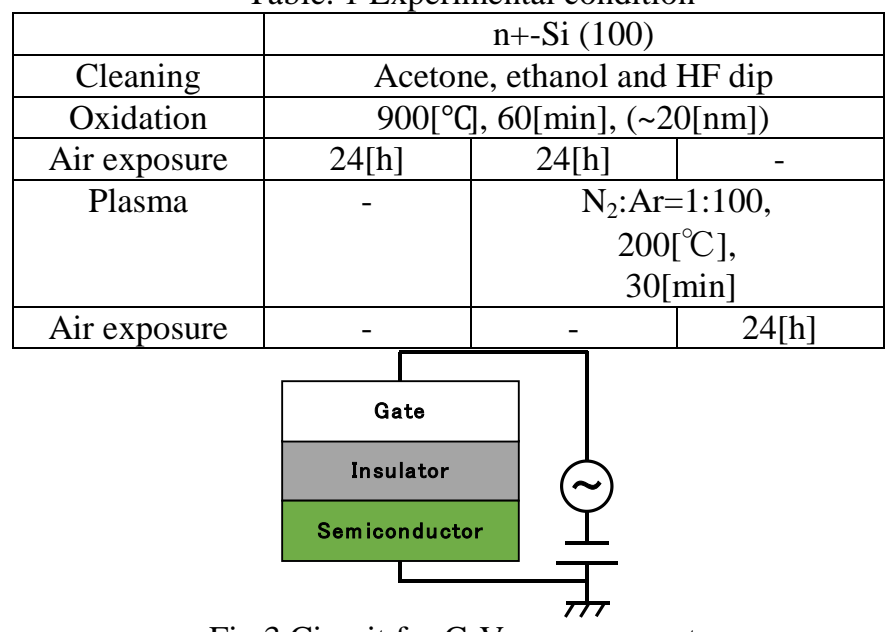

Fig.3 Circuit for C-V measurement

\section{Results and discussion}

\section{A. TDS measurements}

Fig. 4 shows TDS signal of mass number 17 which corresponds to that of $\mathrm{OH}$ groups. It is confirmed that the $\mathrm{OH}$ group on the surface of the oxide film can be removed by plasma nitridation, since the peak near $300\left[{ }^{\circ} \mathrm{C}\right]$ is decreased after the plasma nitridation treatment as in show fig.4 (b). In addition, in the sample subjected to the nitridation treatment, the increase amount of the peak near $300\left[{ }^{\circ} \mathrm{C}\right]$ is small when the air exposure for 1 day is performed as show in fig.4 (c), compared with the sample not subjected to the nitridation treatment show in fig.4 (a). It shows that it is possible to suppress additional carrier traps due to the $\mathrm{OH}$ groups on the gate insulator.

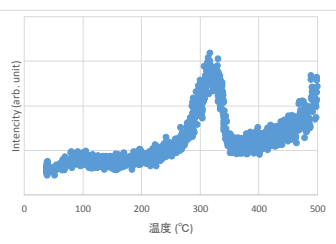

(a) Air exposure for 1day

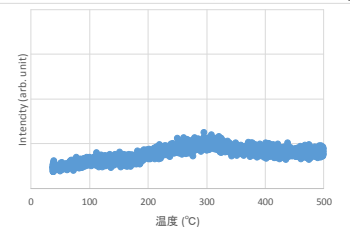

(b) Plasma treatment after air exposure for 1 day

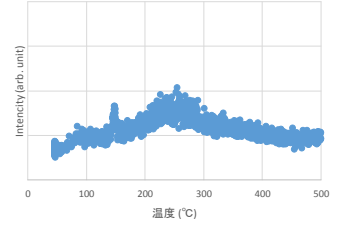

(c) Air exposure for 1day after plasma treatment
Fig.4 TDS measurement 


\section{B. C-V characteristic}

Fig.5 shows the $\mathrm{C}-\mathrm{V}$ characteristic measurement result. Hysteresis occurred when comparing the $\mathrm{C}-\mathrm{V}$ characteristics of the sample exposed to the air after thermal oxidation. This hysteresis is thought to be due to the $\mathrm{OH}$ group generated on the oxide film surface by moisture present in the air. It was confirmed that hysteresis can be greatly reduced at samples subjected to plasma nitridation after exposure to the atmosphere. Furthermore, since no increase in hysteresis was observed when the substrate exposed to the atmosphere after the plasma nitridation, it is considered that generation of new carrier traps could be suppressed by nitridation. It can be seen from the above that the electrical characteristics can be improved by surface nitridation of gate insulator, which would be the interface between organic semiconductor and gate insulator.

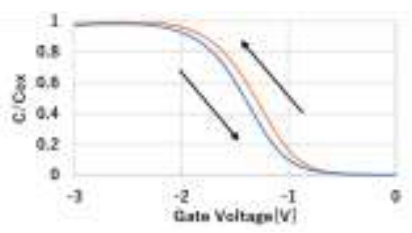

(a) Air exposure for 1day

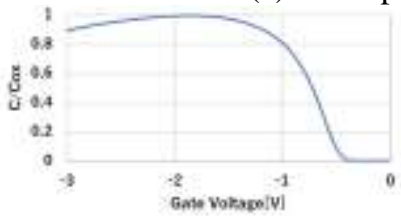

(b) Plasma treatment after air exposure for 1day

Fig.5 C-V characteristics

\section{I-V characteristic}

Fig.6 shows the transistor characteristics obtained by I-V measurement. Compared with the sample which was only subjected to thermal oxidation, transistor characteristics could not be obtained in samples subjected to atmospheric exposure after thermal oxidation. Good transistor characteristics has been obtained in samples nitride after exposure to the atmosphere. In addition, even in samples subjected to air exposure after nitriding treatment, degradation of characteristics was suppressed as compared with thermal oxidation alone, so that generation of new carrier traps can be suppressed by nitridation treatment.

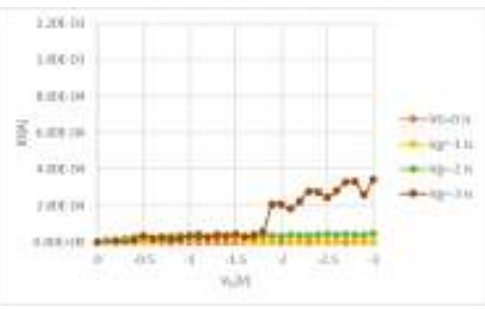

(a) Air exposure for 1day

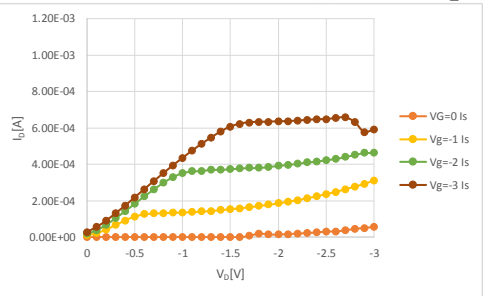

(b) Plasma treatment after air exposure for 1day
Fig.6 I-V characteristic

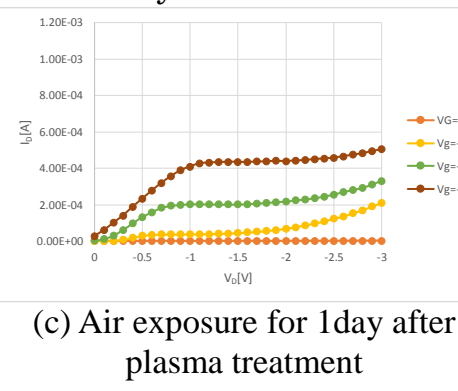
plasma treatment

\section{Iv. Conclusion}

TDS measurement showed that the $\mathrm{OH}$ group on the gate insulator surface can be removed and prevented the occurrence of new carrier traps by nitridation treatment at the surface of oxide films in the OTFT structures. The $\mathrm{C}-\mathrm{V}$ and $\mathrm{I}-\mathrm{V}$ characteristics show that the electric characteristics are improved unless $\mathrm{OH}$ groups are present on the surface of the insulating film by the nitridation

\section{References}

[1] Abdesselam Jedaa, Martin Burkhardt, Ute Zschieschang, Hagen Klauk, Dana Habich, Günter Schmid, Marcus Halik, "The impact of selfassembled monolayer thickness in hybrid gate dielectrics for organic thin-film transistors," in Organic Electronics, 10 1442-1447 (2009)

About Author (s):

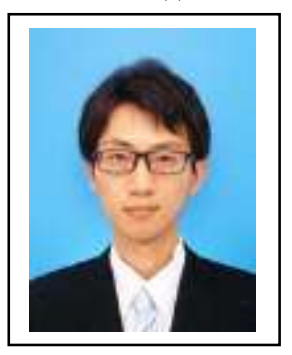

- Master Course, Department of Electrical and Electronic Engineering, graduate school of TUAT (Tokyo univ. of agri. and tech, Japan) 